\title{
Summary Abstract: Elastic and inelastic tunneling characteristics of AIAs/ GaAs heterojunctions
}

\author{
R. T. Collins, J. Lambe, and T. C. McGill \\ California Institute of Technology, Pasadena, California 91125 \\ R. D. Burnham
}

Xerox Corporation, Palo Alto, California 94304

(Received 29 February 1984; accepted 24 April 1984

PACS numbers: $73.40 . \mathrm{Gk}, 73.40 . \mathrm{Lq}$

We report the observation, at low temperatures and bias voltages, of elastic and inelastic tunneling through AlAs barriers sandwiched between two GaAs layers. Previous studies of electronic transport perpendicular to $\mathrm{Al}_{x} \mathrm{Ga}_{1-x} \mathrm{As}$ barriers sandwiched between $\mathrm{GaAs}$ layers ${ }^{1-4}$ have attributed currents passing through the barriers to thermionic emission at temperatures above $150 \mathrm{~K}$ or to tunneling through the $\mathrm{Al}_{x} \mathrm{Ga}_{1-x}$ As barrier at low temperatures, but for bias voltages which were comparable to the barrier height. Two of these studies noted that at low temperatures and low biases more current passed through the barriers than could be accounted for by either of these processes, indicating that some form of leakage may be dominating current transport at low temperatures. ${ }^{1.2}$ In all of these studies the barriers were 300 $\AA$ thick or thicker. The measurements reported here were made on samples with barriers thinner than $300 \AA$ in an effort to enhance the tunneling current at low bias voltages relative to leakage. A more detailed account of most of these measurements is given in Ref. 5 .

The samples used in this study were grown by metalorganic chemical vapor deposition (MOCVD) and by molecular beam epitaxy (MBE). In both cases an $n$-type epitaxial layer of $\mathrm{GaAs}$ was grown on a degenerately doped $n$-type GaAs substrate. A thin layer of AlAs was grown on top of this GaAs layer, followed by a final $n$-type layer of GaAs. In the MOCVD samples the epitaxial $\mathrm{GaAs}$ was doped with Se at $1-5 \times 10^{18} \mathrm{~cm}^{-3}$, and the AlAs layer was either doped $n$ type with $\mathrm{Se}$ or $p$ type with $\mathrm{Mg}$ at approximately $1 \times 10^{18}$ $\mathrm{cm}^{-3}$. The AlAs layers were from 50-300 $\AA$ thick. The MBE samples came from two sources. In both cases the epitaxial GaAs layers were doped with $\mathrm{Si}$ at $1 \times 10^{18} \mathrm{~cm}^{-3}$, and the AlAs was unintentionally doped. The estimated AlAs layer thicknesses were from 50-150 $\AA$. Devices were made on the wafers by defining mesas and fabricating $(\mathrm{Au}, \mathrm{Ge})-\mathrm{Ni}$ contacts on the mesas. Temperature dependent current-voltage $(I-V)$, first derivative $(d I / d V)$, and second derivative $\left(d^{2} I\right)$ $\left.d V^{2}\right)$ spectra were taken on the devices from room temperature to $4.2 \mathrm{~K}$. Zero-bias resistances were obtained from the slope of the $I-V$ curves at $\pm 3 \mathrm{mV}$.

Results of the measurements made on MOCVD grown samples will be discussed first. There was a large difference in the characteristics of the samples, depending upon whether the AlAs barrier was doped $p$ type with $\mathrm{Mg}$ or $n$ type with Se. The $p$-type barriers exhibited increasing resistance as temperature was decreased to $77 \mathrm{~K}$, at which point the $I-V$ curve maintained the same shape down to $4.2 \mathrm{~K}$. The zero-bias resistances of $p$-type barrier samples increased rapidly with barrier width as is seen in Fig. 1. Figure 1 also includes the results of a WKB calculation of the zero-bias resistances expected for various barrier widths. This calculation was based on the technique of Kurtin et al. ${ }^{6}$ Only contributions to the tunneling probability from the AlAs $\Gamma$ points were included. The band offsets and effective masses were taken from Casey and Panish. ${ }^{7}$ Although this model is only presented to give an idea of the orders of magnitude expected for tunneling resistances, the agreement is reasonable for small barrier widths. As the thickness of the barrier is increased, the measured zero-bias resistance does not increase at the rate predicted for tunneling, indicating that for thick AlAs layers, most of the low temperature current is due to some form of leakage. At smaller thicknesses the tunneling

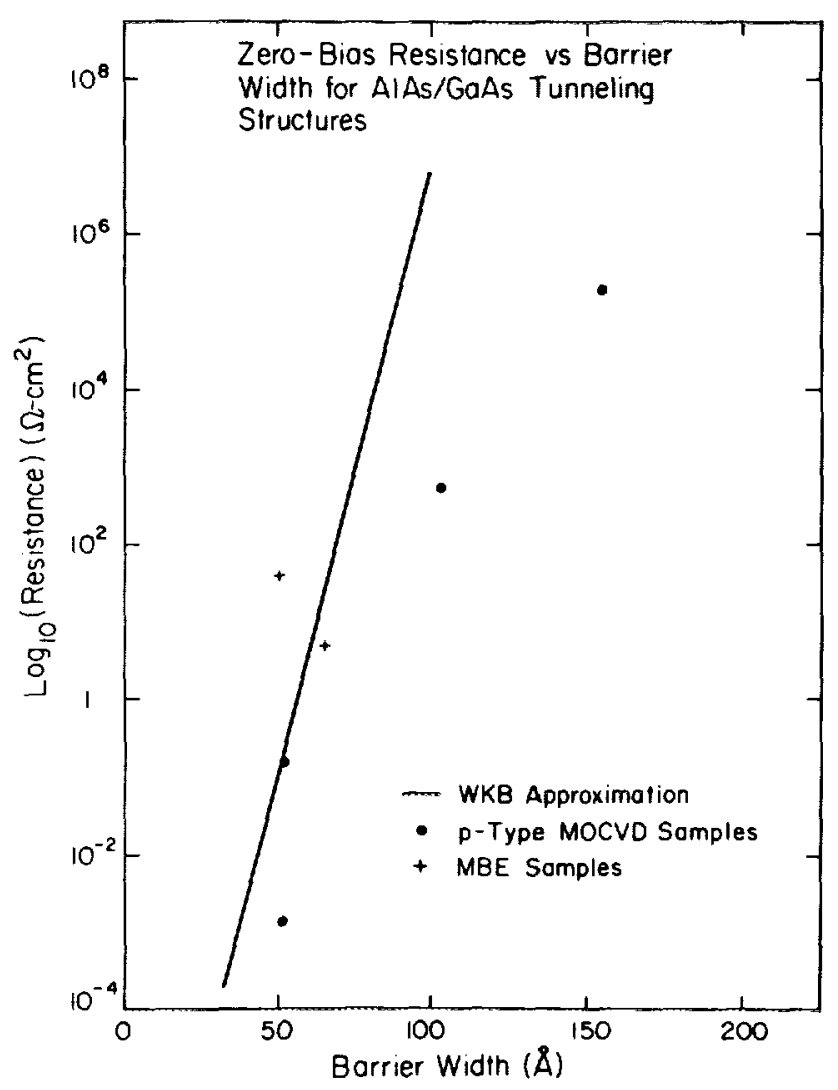

FIG. 1. Zero-bias resistivity at $4.2 \mathrm{~K}$ for MOCVD samples with p-type barriers and for MBE samples which had uniform resistivities between neighboring devices. The solid line is a theoretical calculation of zero-bias resistivity, using a WKB approximation to the tunneling probability. 


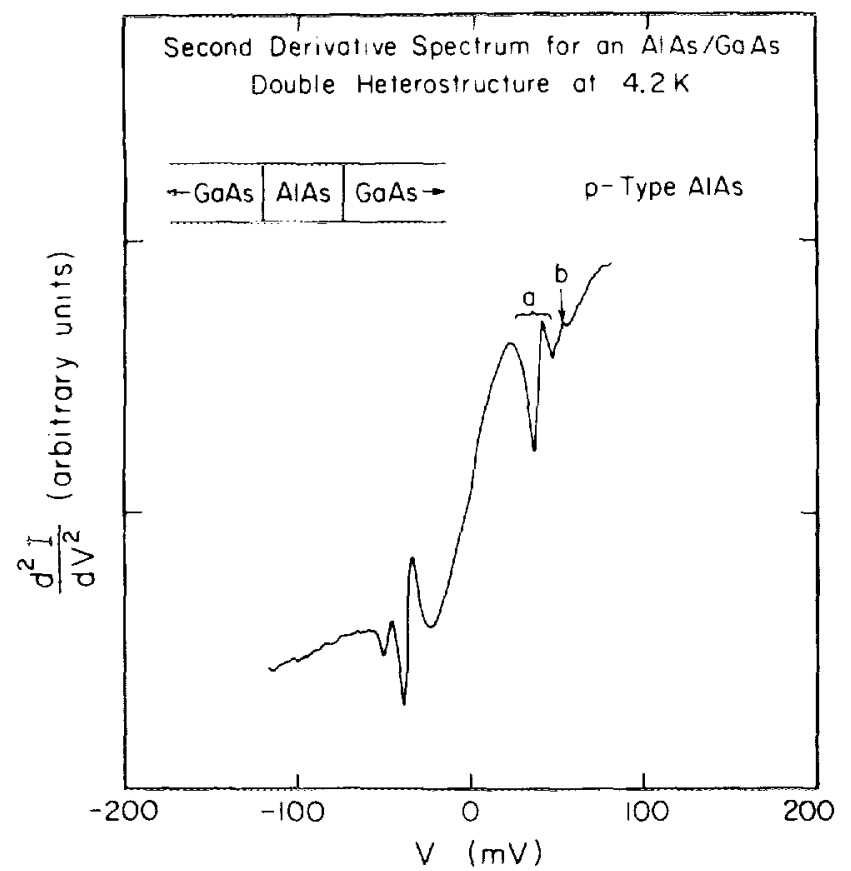

FIG. 2. Second derivative spectrum at $4.2 \mathrm{~K}$ for a $p$-type AlAs barrier sample grown by MOCVD. The layer thickness was approximately $50 \AA$. The structure labeled $|a|$ is attributed to an electronic density-of-states effect caused by electron-optical phonon coupling in the GaAs. The peak labeled (b) is caused by the inelastic excitation of longitudinal optical phonons in the AlAs barrier

current, which decreases exponentially with width, begins to dominate leakage.

When first and second derivatives were taken of the $I-V$ curves of samples with $50 \AA$ thick, $p$-type, AlAs layers, reproducible structure was evident (Fig. 2). Structure [labeled (a)] is present at the GaAs longitudinal optical (LO) phonon energy ( $36 \mathrm{meV}$ ) in both forward and reverse bias in Fig. 2 and is attributed to a density-of-states effect caused by electron-phonon coupling in the degenerate GaAs layers. Similar observations have been made in Schottky barrier tunneling in GaAs. ${ }^{8}$ A peak, labeled (b), is also visible at approximately the AlAs LO phonon energy (50 meV) in the second derivative spectrum. ${ }^{9}$ This peak is associated with the inelastic excitation of longitudinal optical phonons in the AlAs by the tunneling electrons. This is the first observation of these effects in the AlAs/GaAs system. These results were reproducible across a given wafer and were seen in two wafers with barriers of about the same thickness. When the AlAs barrier width increased to $100 \AA$ or greater, no reproducible structure was observed.

When the MOCVD grown AlAs barriers were doped $n$ type, the results were different. The zero-bias resistance changed by less than an order of magnitude as the AlAs layer width was increased from 60 to $250 \AA$. Five peaks were present in the second derivative spectra in both forward and reverse bias for all of the $n$-type barrier samples regardless of width. These peaks did not match those seen in the p-type barrier MOCVD samples. A definitive explanation for the results of measurements on n-type barriers has not been found. A more complete discussion of the differences between $n$ - and $p$-type barrier MOCVD samples and the possible explanations for these differences is given in Ref. 5 .

The zero-bias resistances for devices made from the two MBE grown samples with the thinnest AlAs layers lone from each source) are given in Fig. 1. These resistances are also consistent with the tunneling calculations, although from Fig. 1, it appears that either the MBE or MOCVD barrier widths may be in error by around $10 \AA$. The $I-V$ curves for devices made from these wafers showed approximately the same behavior with temperature as the $p$-type MOCVD barrier samples. Devices made on the two wafers for which resistances are given in Fig. 1 showed some fluctuation from device to device in the zero-bias resistance. The remaining MBE samples studied had barrier widths of $100 \AA$ or greater. The zero-bias resistances of neighboring devices made on these wafers fluctuated by many orders of magnitude. The largest resistances measured for the MBE samples were two or more orders of magnitude larger than the resistances of $p$-type MOCVD samples with the same barrier width. Much smaller fluctuations occurred in the resistivities of devices on the MOCVD grown wafers. The first and second derivative spectra for the MBE wafers did not show any reproducible structure, possibly because they had such large resistances that tunneling currents were beyond the detectable limits of our system. A reason for the large flunctuations in device resistance for the thicker barrier MBE samples has not been found.

Acknowledgments: The authors wish to acknowledge D. L. Smith, C. Mailhiot, R. S. Bauer, T. L. Paoli, and W. Streifer for valuable discussions and L. P. Erickson and G. W. Wicks for providing samples. We are also grateful to $H$. Chung, R. D. Yingling, Jr., F. Endicott, M. Bernstein, M. Mosby, J. Tramontana, J. Walker, A. Alimonday, G. L. Harnagel, and R. Ritter for technical assistance with this work. This work was supported in part by the Office of $\mathrm{Na}$ val Research under Contract No. N00014-82-K-0556.

'D. Delagebeaudeuf, P. Delescluse, P. Etienne, J. Massies, M. Laviron, J. Chaplart, and N. T. Linh, Electron Lett. 18, $85\{1982)$

${ }^{2}$ A. C. Gossard, W. Brown, C. L. Allyn, and W. Wiegmann, J. Vac. Sci. Technol. 20, 694 (1982)

${ }^{3}$ P. M. Solomon, T. W. Hickmott, H. Morkoc, and R. Fischer, Appl. Phys. Lett. 42, 821 (1983).

${ }^{4}$ T. W. Hickmott, P. M. Solomon, R. Fischer, and H. Morkoc, Appl. Phys Lett. $48,90(1984)$.

${ }^{5}$ R. T. Collins, J. Lambe, T. C. McGill, and R. D. Burnham, Appl. Phys. Lett. 44, 532 (1984).

'S. L. Kurtin, T. C. McGill, and C. A. Mead, Phys. Rev. B 3, 3368 (1971).

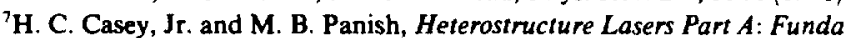
mental Principles (Academic, New York, 1978), p. 192

${ }^{8} \mathrm{~J}$. W. Conley and G. D. Mahan, Phys. Rev. 161, 681 (1967).

${ }^{9} \mathrm{M}$. Ilegems and G. L. Pearson, Phys. Rev. B 1, 1576 (1970) 